ARTICLE

Received 2 Jan 2014 | Accepted 3 Apr 2014 | Published 2 May $2014 \quad$ DOl: 10.1038/ncomms4809

\title{
A generalized non-local optical response theory for plasmonic nanostructures
}

\author{
N.A. Mortensen ${ }^{1,2}$, S. Raza ${ }^{1,3}$, M. Wubs ${ }^{1,2}$, T. Søndergaard ${ }^{4} \&$ S.I. Bozhevolnyi ${ }^{5}$
}

Metallic nanostructures exhibit a multitude of optical resonances associated with localized surface plasmon excitations. Recent observations of plasmonic phenomena at the sub-nanometre to atomic scale have stimulated the development of various sophisticated theoretical approaches for their description. Here instead we present a comparatively simple semiclassical generalized non-local optical response theory that unifies quantum pressure convection effects and induced charge diffusion kinetics, with a concomitant complex-valued generalized non-local optical response parameter. Our theory explains surprisingly well both the frequency shifts and size-dependent damping in individual metallic nanoparticles as well as the observed broadening of the crossover regime from bondingdipole plasmons to charge-transfer plasmons in metal nanoparticle dimers, thus unravelling a classical broadening mechanism that even dominates the widely anticipated short circuiting by quantum tunnelling. We anticipate that our theory can be successfully applied in plasmonics to a wide class of conducting media, including doped semiconductors and low-dimensional materials such as graphene.

\footnotetext{
${ }^{1}$ Department of Photonics Engineering, Technical University of Denmark, DK-2800 Kongens Lyngby, Denmark. ${ }^{2}$ Center for Nanostructured Graphene (CNG), Technical University of Denmark, DK-2800 Kongens Lyngby, Denmark. ${ }^{3}$ Center for Electron Nanoscopy, Technical University of Denmark, DK-2800 Kongens Lyngby, Denmark. ${ }^{4}$ Department of Physics and Nanotechnology, Aalborg University, DK-9220 Aalborg, Denmark. ${ }^{5}$ Department of Technology and Innovation, University of Southern Denmark, DK-5230 Odense, Denmark. Correspondence and requests for materials should be addressed to N.A.M. (email: asger@mailaps.org).
} 
S tudies of transport and wave dynamics in complex and confined geometries ${ }^{1}$ are now bridging several fields ranging from nanoplasmonics ${ }^{2-4}$ and metamaterials ${ }^{5}$ to molecular electronics ${ }^{6}$ and mesocopic quantum transport ${ }^{7,8}$, with, for example, charge carriers responding to externally perturbing fields as well as exhibiting stochastic kinetics and entropic effects such as diffusion ${ }^{9}$. When considering ultrafast responses of optically driven collective plasma oscillations in nanoscale geometries, it is expected that the optical response should exhibit both quantum properties of the electron gas as well as classical diffusion dynamics of the optically induced charge. The coexistence and interplay of quantum and classical effects have profound implications for our understanding of light-matter interactions at the nanoscale, with direct relevance to the emerging field of quantum plasmonics ${ }^{10}$.

The behaviour of plasmon resonances of individual silver metallic nanoparticles (MNPs) ${ }^{11,12}$ and gold MNP dimers ${ }^{13}$ seems to be possible to understand only by invoking quantummechanical effects, that is, quantum electron transitions and quantum tunnelling, respectively. At the same time, one might question the necessity of considering numerous quantum-level transitions in nm-sized NPs (that is, consisting of thousands of atoms and with a size much exceeding the Fermi wavelength) in the case of monomers and the very possible existence of ultrafast tunnelling phenomena (that is, tunnelling currents oscillating at optical frequencies) in the case of dimers. While classical electrodynamics in a Drude local response approximation (LRA) unambiguously fails to explain the observed phenomena, we show that the possibility for semiclassical accounts has not been exhausted.

Linear response theory is inherent to our understanding of situations where matter is subject to externally perturbing fields. Common strategies assume a temporally instantaneous and spatially local response, while nature is rich in examples where underlying degrees of freedom are responsible for a much more complex response. Materials exhibiting frequency dispersion are well known for having complex-valued response functions due to Kramers-Kronig relations that originate from the ubiquitous principle of causality. By contrast, spatial dispersion can usually be neglected and most materials are well treated within LRA. Insulators represent a prime example since the polarization of one particular atom in the crystal is only weakly affected by coupling to neighbouring atoms. Conducting media constitute a clear exception to this picture $e^{14,15}$ and despite the widespread use of LRA approaches, the free carriers may mediate a response over finite distances that cannot necessarily be neglected in a nanoplasmonic context.

In terms of the Maxwell equations, the electrical field in a medium with non-local response is formally governed by

$$
\nabla \times \nabla \times \mathbf{E}(\mathbf{r})=\left(\frac{\omega}{c}\right)^{2} \int d \mathbf{r}^{\prime} \varepsilon\left(\mathbf{r}, \mathbf{r}^{\prime}\right) \mathbf{E}\left(\mathbf{r}^{\prime}\right)
$$

where $\varepsilon\left(\mathbf{r}, \mathbf{r}^{\prime}\right)$ is the non-local response function. This general concept of non-local response of conducting media originates from the competing mechanisms of pressure-driven convective flow of charge as well as disorder- or entropy-driven diffusion of charge ${ }^{14}$. Quite surprisingly, while the literature is rich on discussions of the former effect within hydrodynamic models, the importance of the latter in nanoplasmonic systems remains unexplored, and, according to our knowledge, there is no unifying real-space description applicable to realistic plasmonic nanostructures. Pioneering works focused on pressure-driven convective flow of charge in ideal geometries ${ }^{16-19}$, while the exploration of non-local response in arbitrarily shaped metal nanostructures has only recently been initiated ${ }^{20}$, emphasizing real-space rigorous formulations of semiclassical hydrodynamic equations $^{21}$ and different solution strategies ${ }^{22-26}$. Thus, large blueshifts in nanoscale noble metal plasmonic structures $11,27,28$ have been interpreted in the context of the quantum pressurerelated non-local response $\mathrm{e}^{27,28}$, while quantum confinement ${ }^{11}$ and surface-screening ${ }^{29}$ explanations have also been proposed.

Here we develop a semiclassical generalized non-local optical response (GNOR) theory that incorporates both quantum pressure effects and induced charge diffusion kinetics. We show that the GNOR approach can account for the main features observed in recent optical experiments with plasmonic nanostructures $^{11,13,28,30}$ without accounts for quantized-energy transitions and without invoking quantum tunnelling that should not, as we argue later on, be important at optical frequencies.

\section{Results}

Isotropic and short-range non-local response. We take equation (1) as our starting point, while assuming a generic shortrange isotropic response. Irrespective of the detailed microscopic mechanism behind the non-local response, the wave equation in the metal can then be reframed as 31

$$
\nabla \times \nabla \times \mathbf{E}(\mathbf{r})=\left(\frac{\omega}{c}\right)^{2}\left[\varepsilon_{\mathrm{D}}+\xi^{2} \nabla^{2}\right] \mathbf{E}(\mathbf{r}),
$$

where $\varepsilon_{\mathrm{D}}$ is the Drude dielectric function usually associated with Ohmic local response of the electron gas (possibly generalized to also include interband effects), while the GNOR parameter $\xi$ represents a phenomenological length scale associated with the short-range non-local correction to the local response Drude part. Importantly, the GNOR parameter accounts for mechanisms of very different origins that may compete or play in concert, while causing the same Laplacian-type correction to the LRA. For example, both convection and diffusion can lead to spatial dispersion in conducting media ${ }^{14}$. Within LRA, the induced charge density $\Delta n$ is a delta function at the surface of the metal and diffusion will naturally smear this charge density with the short-time dynamics characteristic for pure diffusion (Fig. 1c). Also, convection tends to spread the charge density and within the common hydrodynamic Drude model, convection maps directly to the form of equation (2), that is, with a Laplacian correction to the local response Drude part ${ }^{24}$. In the following, we treat both dynamical effects on an equal footing by considering both propagating longitudinal pressure waves (in a hydrodynamic model) and diffusion (in convection-diffusion model). The main result of our analysis is the following expression for the GNOR parameter:

$$
\xi^{2} \simeq \frac{\beta^{2}}{\omega^{2}}-i \frac{D}{\omega}
$$

where $\beta \propto v_{\mathrm{F}}$ is a characteristic velocity associated with pressure waves in the electron gas ( $v_{\mathrm{F}}$ being the Fermi velocity) while $D$ is the diffusion constant for the charge-carrier diffusion. The former is already known to cause frequency shifts (blueshifts) $^{17,21,22}$, while the latter turns out to cause line broadening, that is the GNOR parameter $\xi$ is in general a complex-valued quantity. As also anticipated from more general discussions $^{14}$, our rigorous semiclassical treatment shows that non-local effects may manifest themselves over distances greatly exceeding atomic dimensions and become comparable to characteristic structure dimensions, such as the radius $R$ of a nanoparticle (Fig. 1a) or the gap distance $g$ in a dimer (Fig. 1b). As a main result, we show that the GNOR even dominates more pure quantum-mechanical effects in the electromagnetic response at optical frequencies, such as the anticipated effect of quantum-mechanical tunnelling currents in dimers with sub-nanometre gaps (Fig. 1b). 
a

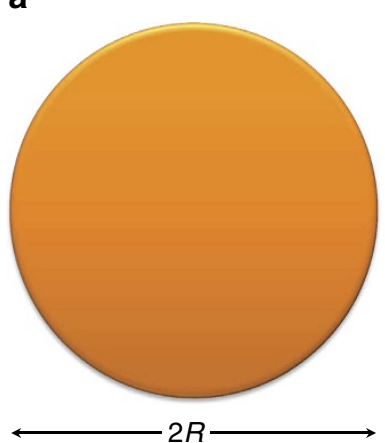

b
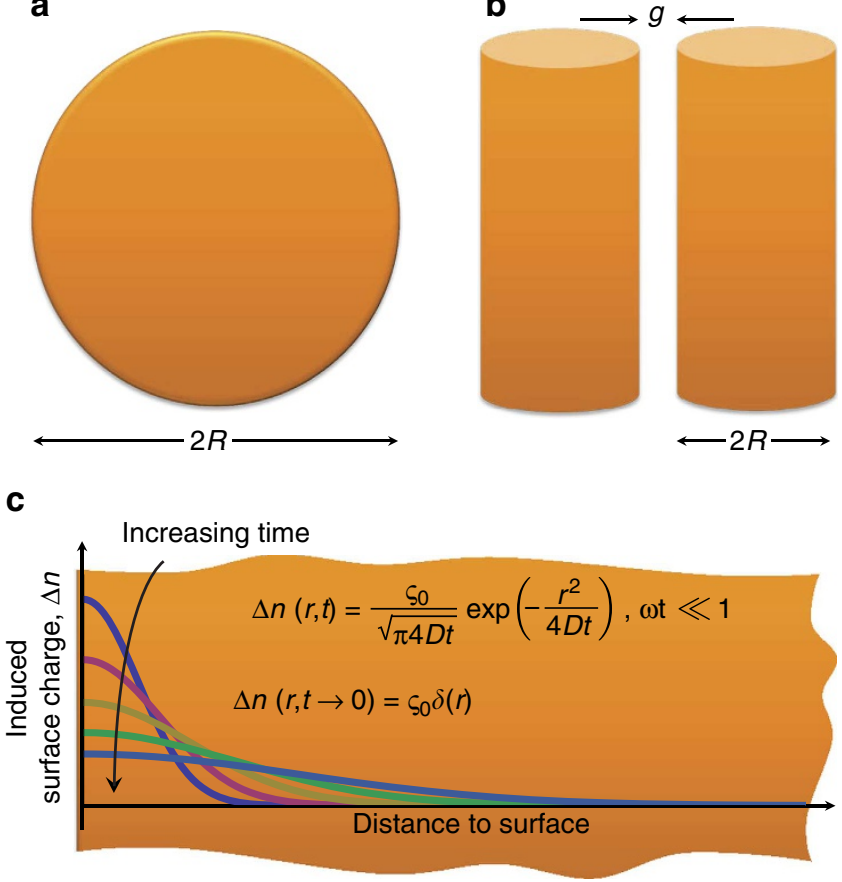

Figure 1 | Nanoplasmonic monomer and dimer structures. (a) Spherical particle of radius $R$, known to experimentally exhibit size-dependent damping 37,38 and resonant shifts ${ }^{11,12,28}$. (b) Nanowires of radius $R$ arranged parallel to each other with a dimer gap $g$. Dimers are known experimentally to exhibit gap size-dependent broadening and shifts of hybridized plasmonic resonances ${ }^{13,30}$. (c) Diffusive temporal spreading of an initially pure surface charge $s_{0}$ into the metal volume (orange-shaded area) of a plasmonic nanoparticle. By accounting for diffusion of charge, the GNOR theory can explain size-dependent broadening and shifts of nanoparticle resonances, as well as gap size-dependent broadening and shifts for dimer resonances. Unlike other theories (including the $\mathrm{QCM}^{52}$ ), our GNOR theory does not invoke quantum tunnelling to explain gap size-dependent spectral broadening.

Semiclassical response models. We consider the standard equation-of-motion for an electron in an external electrical field subject to the continuity equation. The common LRA simply neglects effects of quantum pressure as well as the diffusion contributions to the induced currents. We address these two aspects in turn and finally discuss how they play in concert to result in a complex-valued GNOR parameter.

Within the hydrodynamic model (including quantum pressure, but neglecting diffusion), the response is governed by a generalized constitutive equation ${ }^{21,22}$

$$
\frac{\beta^{2}}{\omega(\omega+i \gamma)} \boldsymbol{\nabla}\left(\boldsymbol{\nabla} \cdot \mathbf{J}_{\text {conv }}\right)+\mathbf{J}_{\text {conv }}=\sigma_{\mathrm{D}} \mathbf{E}
$$

where $\sigma_{\mathrm{D}}$ is the usual Drude conductivity. Within Thomas-Fermi theory, $\beta^{2}=(3 / 5) v_{\mathrm{F}}^{2}$ and $\gamma=1 / \tau$ is the damping rate also present in the Drude theory.

Taking the opposite standpoint (including diffusion, while neglecting quantum pressure), linearization of the problem gives (Supplementary Note 1)

$$
\frac{D}{i \omega} \boldsymbol{\nabla}\left(\boldsymbol{\nabla} \cdot \mathbf{J}_{\mathrm{dif}}\right)+\mathbf{J}_{\mathrm{dif}}=\sigma_{\mathrm{D}} \mathbf{E}
$$

as also derived recently in the context of metamaterial wire media $^{32}$. Our key observation is that this result is mathematically similar to equation (4), while the different physical origins cause different prefactors for the non-local correction to Ohm's law.
Turning to the Maxwell wave equation, the above non-local $\boldsymbol{\nabla}(\boldsymbol{\nabla} \cdot \mathbf{J})$ corrections to Ohm's law can be rewritten as a Laplacian correction to the Drude dielectric function ${ }^{24,31}$, as anticipated in equation (2). The convection and diffusion components of the current are of the same mathematical form and subject to the same boundary conditions. Thus, in the linear response the considered non-local contributions add up (as in semiconductor drift-diffusion theory), as confirmed by linearizing the full hydrodynamic diffusion-convection problem (Supplementary Note 1), revealing how both quantum and classical kinetic effects can play in concert contributing to the non-local response. Therefore, we arrive at equation (2) with

$$
\xi^{2}=\frac{\beta^{2}}{\omega(\omega+i \gamma)}-i \frac{D}{\omega}=\frac{\beta^{2}+D(\gamma-i \omega)}{\omega(\omega+i \gamma)},
$$

which becomes equation (3) when neglecting damping. Note that for higher $\omega$, diffusion becomes relatively more important compared with convection. Another important practical observation is that diffusion effectively causes the following modification of the non-local $\beta$-parameter appearing in prior hydrodynamic work: $\beta^{2} \rightarrow \beta^{2}+D(\gamma-i \omega)$. We explicitly neglect electron spill-out and the possible existence of associated quantum-tunnelling phenomena (see also Supplementary Note 2 ). Consequently, boundary conditions remain unchanged in the presence of diffusion $(\mathbf{n} \cdot \mathbf{J}=0$ on the metal surfaces so that no electrons escape the metal volumes). Thus, existing numerical schemes and methods ${ }^{22-26}$ can readily be exploited to implement the GNOR approach for various plasmonic configurations.

The diffusion constant is generally interlinked with other transport parameters such as the scattering time, that is, $D \propto \tau=\gamma^{-1}$. For $\omega \gg \gamma$, we thus recover equation (3) for the GNOR parameter that now explicitly exposes the two competing length scales that were previously discussed only qualitatively ${ }^{14}$, that is, the convection length $\beta / \omega$ on the one hand and the diffusion length $\sqrt{D / \omega}$ on the other hand. The damping associated with the latter is an important new finding that turns out to be crucial when approaching the nanoscale. Diffusion degrades plasmonic excitations, providing an additional broadening mechanism that, mathematically, is enacted by an imaginary contribution to $\xi^{2}$.

Validity domain. For classical gases (such as dilute plasmas, electrolytes and weakly doped semiconductors), the velocity distribution is governed by Maxwell-Boltzmann statistics and $D$ is proportional to the temperature, as given by the Einstein relation. For metals on the other hand, Fermi-Dirac statistics implies a narrow transport velocity distribution ${ }^{14}$ with a characteristic velocity $v_{\mathrm{F}}$. As a result, the diffusion constant is simply $D \simeq v_{\mathrm{F}}^{2} \tau$, corresponding to a mean-free path of $\ell=v_{\mathrm{F}} \tau$. We point out that our diffusive model is valid for structural dimensions exceeding the mean-free path that in pure single crystals ${ }^{33}$ can be of the order of $100 \mathrm{~nm}$ for $\mathrm{Ag}$ and $\mathrm{Au}$, down to $\sim 3 \mathrm{~nm}$ for $\mathrm{Na}$ (see Supplementary Table 1). Moreover, in realistic plasmonic nanostructures, $\ell$ depends on actual materialprocessing conditions, becoming shorter than in single-crystalline bulk metals. This enlarges the validity domain of a diffusion description to include structures with dimensions of only a few nanometres. For even smaller dimensions, electrons will move ballistically between the surfaces of the structure, and surface scattering might become important. For metals, equation (6) simplifies to $\xi^{2}=\frac{v_{\mathrm{F}}^{2}}{\omega^{2}}\left(\frac{3}{5}-i \frac{\omega}{\gamma}\right)+\mathcal{O}(\gamma / \omega)$. This result leads to an important insight into the interplay of different broadening mechanisms: the lower the Ohmic loss and absorption, the more important is the non-local response due to long-range diffusion of the induced charge. Re-introducing the diffusion constant 
and the $\beta$-parameter we arrive at equation (3) that holds if the mean-free path significantly exceeds the convective length, that is, $\ell \gg v_{\mathrm{F}} / \omega$. The existence of spatial dispersion in homogenous media, as for example, appearing within the common hydrodynamic Drude model, can be derived from higher-level descriptions such as the Boltzmann equation or the randomphase approximation (RPA). Beyond hydrodynamics, confined structures with broken translational invariance constitute a largely unexplored territory, with few attempts dealing with RPA aspects of localized plasmon resonances ${ }^{34}$. In support of our prediction of diffusive broadening, recent RPA studies reveal an increased plasmon linewidth associated with Landau damping ${ }^{35}$, that is, electron-hole pair excitation near the surface of nanostructures as also observed in studies based on timedependent density functional theory (TD-DFT) ${ }^{36}$.

We provide in the following two key examples of the GNOR approach, demonstrating that the interplay of quantum pressure and diffusion has a remarkable impact on the optical response of plasmonics nanostructures and solving long-standing open problems.

Size-dependent damping. In general, the hydrodynamic corrections give a blueshift of resonances as the characteristic dimensions are reduced ${ }^{17,22,27,28}$, which is in qualitative accordance with recent optical spectroscopy on gold-particle gap structures ${ }^{27}$ and electron-energy loss spectroscopy (EELS) studies of silver nanoparticles ${ }^{11,28}$. With the complex-valued GNOR parameter $\xi$ at hand, we now anticipate the blueshift to occur along with broadening of the resonant response when decreasing characteristic structure dimensions. In the case of a spherical particle, the blueshift has a $\beta / R$ dependence ${ }^{28}$, leading us to foresee that the line-broadening scales as $1 / R$ as well. In the quasi-static limit $(\lambda \gg R)$, one can straightforwardly work out the complex-valued resonance frequency $\omega=\omega^{\prime}+i \omega^{\prime \prime}$ by considering the polarizability pole (Supplementary Note 3 ). As usual, the real part $\omega^{\prime}$ gives the surface plasmon resonance frequency, while the imaginary part $\omega^{\prime \prime}$ is related to the resonance linewidth. For simplicity, we consider the case of a particle in vacuum with no interband effects and find (to second order in $1 / R$ )

$$
\begin{gathered}
\omega^{\prime} \simeq \frac{\omega_{\mathrm{p}}}{\sqrt{3}}+\frac{\sqrt{2} \beta}{2 R}, \\
\omega^{\prime \prime} \simeq-\frac{\gamma}{2}-\frac{\sqrt{6}}{24} \frac{D \omega_{\mathrm{p}}}{\beta R} .
\end{gathered}
$$

It transpires clearly that the $1 / R$ size-dependent non-local effects are present in both the resonance frequency and linewidth. It should be emphasized that, until now, line shifts have been explained by non-local response (and competing theories), whereas the line broadening was 'put in by hand'. Here by using the GNOR theory we have arrived at a unified explanation of both experimentally observed phenomena by non-local effects. Line broadening has been seen experimentally in the extinction of small particles ${ }^{37-41}$ and EELS measurements on plasmons in thin nanowires and bow-tie antennas have also revealed plasmon losses exceeding the expectations based on bulk-damping parameters ${ }^{42,43}$. In the literature such line broadening has often been phenomenologically accounted for by a size-dependent

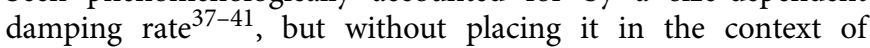
non-local semiclassical equations of motion.

The phenomenology introduced by Kreibig ${ }^{37,38}$ describes the linewidth broadening by introducing a size-dependent correction to the damping rate: $\gamma \rightarrow \gamma+A v_{\mathrm{F}} / R$. Equipped with the GNOR theory, one does not need to assume the $1 / R$ dependence: it comes immediately out as a consequence of the GNOR correction to the dipolar sphere polarizability. By comparison with the Kreibig model, we formally find that $A=\sqrt{\frac{1}{24}} \frac{D \omega_{\mathrm{p}}}{\beta v_{\mathrm{F}}}$. For metals this implies that $A \sim \omega_{\mathrm{p}} \tau / 4$. Use of bulk values for noble metals (see Supplementary Table 1) would estimate a too high $A$ parameter compared with experiments where for spheres $A$ is found to be of the order unity ${ }^{37,38}$.

So far, we have assumed that the transport time is given by the bulk relaxation time $\tau_{0}$. However, in the experiments dealing with plasmonic nanostructures, it has been found that one has to increase the collision frequency ${ }^{44,45}$ or the imaginary part of permittivity ${ }^{46}$ by several times as compared with the bulk metal (gold) parameters for the simulations to better correspond to the experimental observations. This size-independent correction factor was ascribed to the influence of the surface scattering and grain boundary effects in nanostructures ${ }^{44-46}$. In our case, we can simply introduce the characteristic relaxation time $\tau_{\mathrm{s}}$ associated with these effects, which can be estimated from the condition that $A \sim 1$ as $\tau_{\mathrm{s}} \sim 4 / \omega_{\mathrm{p}}$.

With the above refinement at hand we can demonstrate using numerical simulations (Fig. 2) that, in agreement with equations (7) and (8), the hydrodynamic response causes a blueshift (Fig. 2a), whereas the diffusion causes an additional broadening (Fig. 2b). In the latter figure, it is also shown that a similar resonance broadening (but not the blueshift) is predicted by the LRA with additional Kreibig damping.

Our real-space non-local wave equation (equation (2)) along with the GNOR parameter (equation (3)), which unravels the fundamental link between diffusive broadening and the Kreibiglike surface scattering thereby enables one to solve a long-

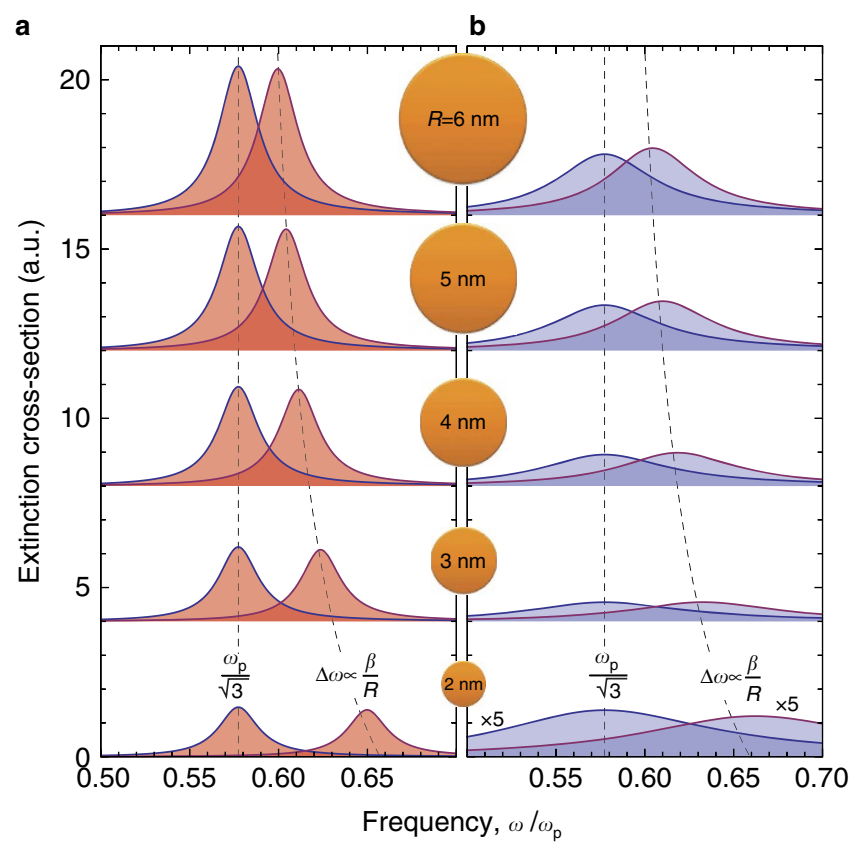

Figure 2 | Extinction cross-section for the dipole resonance in a metal sphere. The sphere radius $R$ is varied from 2 to $6 \mathrm{~nm}$. (a) LRA ( $\beta=D=0)$ versus hydrodynamic non-local response $(\beta \neq 0, D=0)$, showing a blueshift $\Delta \omega$ (indicated by blueshifting-dashed lines) with respect to the common local response resonance at $\omega_{\mathrm{p}} / \sqrt{3}$ (indicated by vertical-dashed lines). (b) LRA (including $1 / R$ Kreibig damping ${ }^{38}$ with the experimentally relevant value of $A=1)$ versus the GNOR model $(\beta \neq 0, D \neq 0)$, with the latter capturing both the line shift and broadening. The metal parameters for sodium are used ${ }^{49,38}: \omega_{\mathrm{p}}=5.89 \mathrm{eV}, \gamma=0.16 \mathrm{eV}, v_{\mathrm{F}}=1.05 \times 10^{6} \mathrm{~ms}^{-1}$, $\beta=0.81 \times 10^{6} \mathrm{~ms}^{-1}, D=2.04 \times 10^{-4} \mathrm{~m}^{2} \mathrm{~s}^{-1}$, and $A=1$. 
standing open problem: surface-related scattering can now be computationally accounted for also in complex-shaped geometries beyond that of spherical symmetry and low radius of curvature.

Nanowire dimers. Plasmonic dimers (Fig. 1b) are rich on hybridization phenomena as the gap distance $g$ is reduced ${ }^{47}$ and non-local hydrodynamic effects on both hybridization and field enhancement have been anticipated ${ }^{22}$. To elucidate the diffusion contribution to non-local effects, we may consider dimers of nanowires where the nanowire radius $R$ itself is too large to cause either non-local hydrodynamic effects or increased damping of the Kreibig kind. Nevertheless, we expect that the dimer would exhibit non-local effects once the dimer gap distance $g$ turns comparable in magnitude to $\xi$, resulting in additional broadening in the vicinity of the gap as $v_{\mathrm{F}} / g$ increases. With our present formalism, this can now be quantified without any need to invoke ad hoc assumptions specifically for dimers.

Although diffusion is of a classical origin, the discussion of its effect in dimers ties up with very recent experiments on dimers in the quantum-tunnelling regime ${ }^{13}$. Ab initio approaches show a crossing from the classical hybridization of localized surface plasmon resonances to tunnelling-mediated charge-transfer plasmons (CTPs) ${ }^{36,48-50}$. Being able to push experiments into this intriguing regime $\mathrm{e}^{13,30,51}$, commonly associated with expectations of quantum physics, leaves an open question: Can this regime be adequately described with semiclassical models? While non-local response within the hydrodynamic semiclassical model has been found unsuccessful in explaining features from TD-DFT ${ }^{4-50}$, there have been phenomenological attempts of classically modelling the crossover regime. The 'quantumcorrected model' (QCM) ${ }^{52}$ adds an artificial conducting and lossy material in the gap to mimic short-circuiting currents associated with quantum tunnelling. While apparently successful in qualitatively fitting results of $a b$ initio simulations ${ }^{49,50,52}$, the model raises concerns regarding its physical foundation. The well-established understanding of mesoscopic quantum electron transport ${ }^{7}$ is that the tunnelling through the classically forbidden gap region is elastic (ballistic transport) while energy relaxation takes place inside the metallic contact regions. Opposite to that, the artificial gap material introduced in the QCM causes dissipation within the gap, while there is no associated relaxation occurring on the metal sides of the junction.

While $a b$ initio works emphasize tunnelling ${ }^{52,49,50}$, recent experiments on dimers ${ }^{13,30}$ do not offer explicit evidence that the broadening is associated with quantum tunnelling. The formation of a sub-nanometre gap is evident from the observed DC voltagedriven tunnelling current $^{13}$, while there is no explicit confirmation of AC tunneling currents caused by the optical driving. Optical rectification phenomena are possible by photonassisted inelastic tunnelling currents ${ }^{53}$, while earlier time-resolved elastic-tunnelling experiments have reported tunnelling RC times (i.e. the characteristic time scale of the equivalent resistorcapacitor circuit) in the picosecond range ${ }^{54}$, thus suggesting a suppression of optical frequency tunneling currents that would take place in femtoseconds. This apparently makes quantumtunnelling dynamics too slow and a less likely mechanism to explain the broadening of dimer modes at optical frequencies. Applying the GNOR framework, we demonstrate in the following that the diffusion offers a strong competing damping mechanism. In fact, for fast driving of the junction ${ }^{55}$, diffusion may completely dominate the dissipation of the dimer junction as we illustrate by a circuit analysis (see Supplementary Note 2).

The diffusion-driven damping occurs right inside the surface of the metals (not in the gap), becoming progressively more pronounced for smaller gaps and vanishing for large gaps. To exemplify this in detail, we revisit recent non-local (hydrodynamic) simulations ${ }^{49}$, while making sure to account for the diffusive broadening as well by use of the complex-valued GNOR parameter (Fig. 3). For relatively large gaps, one observes the bonding-dipole plasmon (BDP) along with higher-order modes known to appear below the plasma frequency within the LRA ${ }^{56}$ (for a larger radius this becomes particularly clear, see Supplementary Fig. 1). As the gap is reduced to the non-local regime $g \lesssim v_{\mathrm{F}} / \omega$, as considered in Fig. 3 for $R=4.9 \mathrm{~nm}$, resonances are slightly blueshifted with respect to the LRA result ${ }^{22}$. When the gap shrinks further, progressively stronger hybridization $^{47}$ and accordingly larger BDP redshifts are clearly seen. At the same time, the BDP is gradually suppressed owing to the increasing role of diffusion as the contact point, $g=0$, is approached. This is in strong contrast to predictions from both the LRA $^{56}$ (with even diverging field enhancement) and from previous non-local theories that treated the $\beta$-parameter realvalued $^{22,49,50,57}$ (see Supplementary Fig. 1). As we enter the contact regime, the BDP fades away, vanishing completely for $g<0$. For touching wires, the CTP appears, whose resonance blueshifts and grows in strength for larger wire overlaps. We note that, for $g \sim 0$, the diffusive broadening is so strong that only

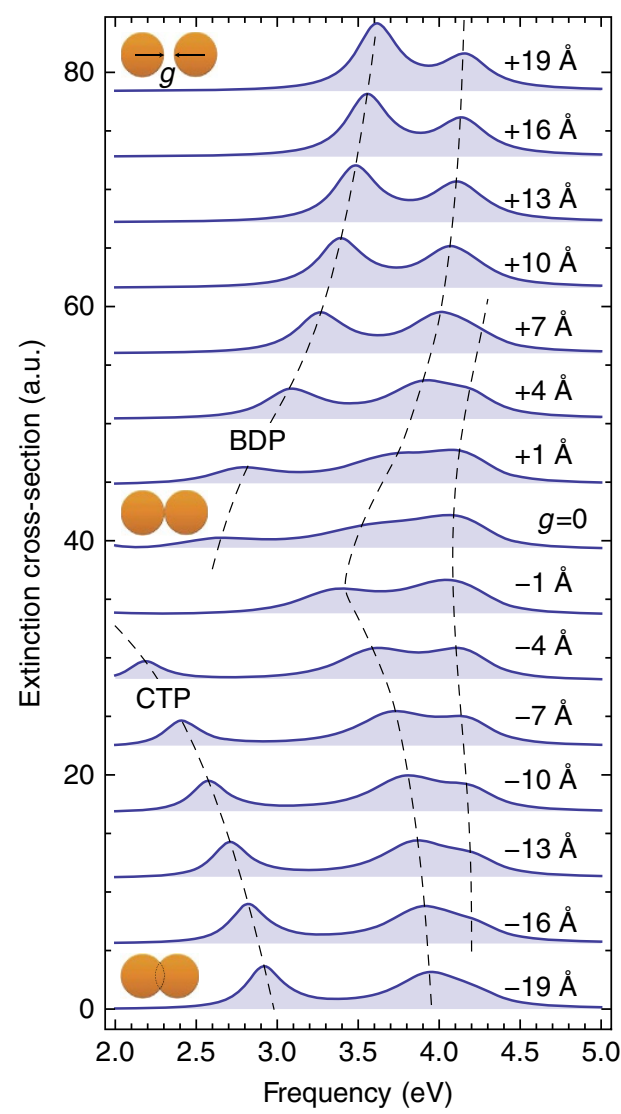

Figure 3 | Extinction cross-section for a nanowire dimer with a sub-nanometre gap within the GNOR model. The radius of the sodium wires is $R=4.9 \mathrm{~nm}$ with the gap $g$ varying from -19 to $+19 \AA$. Progressively stronger hybridization occurs as the gap narrows, with both a clear redshift and broadening of the BDP. As the gap closes, the CTP develops and blueshifts as the wires start to overlap. Higher-order modes (also indicated by dashed lines) exhibit hybridization and broadening too. The diffusion with constant $D=1.36 \times 10^{-4} \mathrm{~m}^{2} \mathrm{~s}^{-1}$ causes GNOR spectra in accordance with TD-DFT calculations ${ }^{49}$ and in overall agreement with the broadening observed experimentally ${ }^{13,30}$. 
higher-order modes persist (as the induced surface charge is located away from the contact point), while both BDP and the CTP are strongly suppressed. This makes a discussion on their possible coexistence problematic ${ }^{51}$. Finally, we note that in the anticipated tunnelling regime the extinction spectra are strongly broadened by the complex non-local response. In fact, our semiclassical approach is in remarkable agreement with the TD-DFT results ${ }^{48-50}$, with the diffusion contribution being responsible for 'repairing' the apparent incompatibility of TD-DFT calculations and earlier hydrodynamic predictions ${ }^{49,50}$. Our semiclassical GNOR theory thereby pinpoints induced charge diffusion as the dominant broadening mechanism in recent EELS and optical experiments on plasmonic dimers ${ }^{13,30}$, thus challenging tunnelling-current interpretations for which the phenomenological QCM was constructed.

\section{Discussion}

In this article we have presented a semiclassical (GNOR) approach that is offering a long-sought unification of non-local response mechanisms having both quantum-mechanical and classical origins. The GNOR theory places established observations of size-dependent damping into the context of non-local response and offers an accurate classical explanation of spectral broadening in MNP monomers and dimers without invoking quantummechanical tunnelling, whose efficiency at optical frequencies is questionable. We have so far considered degenerate electron systems such as metals, where screening is strong and non-local effects manifest themselves in the nanometre to sub-nanometre regime. In the search for a new mesoscopic regime, where plasmons potentially exhibit both semiclassical dynamics and quantum effects, low-density-doped semiconductors and tunable low-dimensional materials (including the graphene family of twodimensional materials) appear attractive $\mathrm{e}^{58}$. Quantum light-matter interactions ${ }^{59}$ and non-local response ${ }^{60}$ were already considered for graphene plasmonics, and, for such non-degenerate systems, our theory anticipates temperature-dependent non-local response that might lead to novel non-local effects accessible via experimental observations.

\section{Methods}

The optical response of dimers was obtained by solving the non-local wave equation with the aid of a commercially available finite-element method. The numerical code is an extension to the COMSOL 4.2a RF Module, which incorporates non-local hydrodynamic effects in the optical response of arbitrarily shaped nanoplasmonic structures, possessing one dimension with translational symmetry. The code is freely available from http://www.nanopl.org and the implementation, testing and performance are reported elsewhere ${ }^{22,24}$. Prior results on dimers ${ }^{49}$ were obtained with the same code, while only utilizing a real-valued $\beta$-parameter. All numerical results can alternatively be calculated with the aid of a non-local boundary-element method ${ }^{26}$. Similarly, the complex-valued GNOR parameter can be substituted in non-local transformation optics approaches originally developed with a real-valued $\beta$-parameter in mind ${ }^{57}$.

\section{References}

1. Van Rossum, M. \& Nieuwenhuizen, T. Multiple scattering of classical waves: microscopy, mesoscopy, and diffusion. Rev. Mod. Phys. 71, 313-371 (1999).

2. Schuller, J. A. et al. Plasmonics for extreme light concentration and manipulation. Nat. Mater. 9, 193-204 (2010).

3. Gramotnev, D. K. \& Bozhevolnyi, S. I. Plasmonics beyond the diffraction limit. Nat. Photon. 4, 83-91 (2010).

4. Gramotnev, D. K. \& Bozhevolnyi, S. I. Nanofocusing of electromagnetic radiation. Nat. Photon. 8, 14-23 (2014).

5. Engheta, N. Circuits with light at nanoscales: optical nanocircuits inspired by metamaterials. Science 317, 1698-1702 (2007).

6. Nitzan, A. \& Ratner, M. Electron transport in molecular wire junctions. Science 300, 1384-1389 (2003).

7. Datta, S. Electronic Transport in Mesoscopic Systems (Cambridge University Press, 1995).

8. Beenakker, C. W. J. Random-matrix theory of quantum transport. Rev. Mod. Phys. 69, 731-808 (1997).
9. Burada, P. S., Hänggi, P., Marchesoni, F., Schmid, G. \& Talkner, P. Diffusion in confined geometries. Chemphyschem 10, 45-54 (2009).

10. Tame, M. S. et al. Quantum plasmonics. Nat. Phys. 9, 329-340 (2013).

11. Scholl, J. A., Koh, A. L. \& Dionne, J. A. Quantum plasmon resonances of individual metallic nanoparticles. Nature 483, 421-427 (2012).

12. Ouyang, F., Batson, P. \& Isaacson, M. Quantum size effects in the surfaceplasmon excitation of small metallic particles by electron-energy-loss spectroscopy. Phys. Rev. B 46, 15421-15425 (1992).

13. Savage, K. J. et al. Revealing the quantum regime in tunnelling plasmonics. Nature 491, 574-577 (2012).

14. Landau, L. D., Lifshitz, E. M. \& Pitaevskii, L. P. Course on Theoretical Physics 2nd edn, Vol. 8 (Butterworth Heinemann, 1984).

15. Boardman, A. Electromagnetic Surface Modes (John Wiley and Sons, 1982).

16. Fuchs, R. \& Kliewer, K. L. Optical properties of an electron gas: further studies of a nonlocal description. Phys. Rev. 185, 905-913 (1969).

17. Ruppin, R. Optical properties of a plasma sphere. Phys. Rev. Lett. 31, 1434-1437 (1973).

18. Dasgupta, B. B. \& Fuchs, R. Polarizability of a small sphere including nonlocal effects. Phys. Rev. B 24, 554-561 (1981).

19. García de Abajo, F. J. Nonlocal effects in the plasmons of strongly interacting nanoparticles, dimers, and waveguides. J. Phys. Chem. C 112, 17983-17987 (2008).

20. McMahon, J. M., Gray, S. K. \& Schatz, G. C. Nonlocal optical response of metal nanostructures with arbitrary shape. Phys. Rev. Lett. 103, 097403 (2009).

21. Raza, S., Toscano, G., Jauho, A.-P., Wubs, M. \& Mortensen, N. A. Unusual resonances in nanoplasmonic structures due to nonlocal response. Phys. Rev. B 84, 121412(R) (2011).

22. Toscano, G., Raza, S., Jauho, A.-P., Mortensen, N. A. \& Wubs, M. Modified field enhancement and extinction in plasmonic nanowire dimers due to nonlocal response. Opt. Express 20, 4176-4188 (2012).

23. Hiremath, K. R., Zschiedrich, L. \& Schmidt, F. Numerical solution of nonlocal hydrodynamic Drude model for arbitrary shaped nano-plasmonic structures using Nédélec finite elements. J. Comp. Phys. 231, 5890-5896 (2012).

24. Toscano, G. et al. Nonlocal response in plasmonic waveguiding with extreme light confinement. Nanophotonics 2, 161-166 (2013).

25. Luo, Y., Fernández-Domínguez, A. I., Wiener, A., Maier, S. A. \& Pendry, J. B. Surface plasmons and nonlocality: a simple model. Phys. Rev. Lett. 111, 093901 (2013).

26. Yan, W., Mortensen, N. A. \& Wubs, M. Green's function surface-integral method for nonlocal response of plasmonic nanowires in arbitrary dielectric environments. Phys. Rev. B 88, 155414 (2013).

27. Ciraci, C. et al. Probing the ultimate limits of plasmonic enhancement. Science 337, 1072-1074 (2012).

28. Raza, S. et al. Blueshift of the surface plasmon resonance in silver nanoparticles studied with EELS. Nanophotonics 2, 131-138 (2013).

29. Monreal, R. C., Antosiewicz, T. J. \& Apell, S. P. Competition between surface screening and size quantization for surface plasmons in nanoparticles. New J. Phys. 15, 083044 (2013).

30. Scholl, J. A., Garcia-Etxarri, A., Koh, A. L. \& Dionne, J. A. Observation of quantum tunneling between two plasmonic nanoparticles. Nano Lett. 13, 564-569 (2013).

31. Mortensen, N. A. Nonlocal formalism for nanoplasmonics: phenomenological and semi-classical considerations. Phot. Nanostr. 11, 303-309 (2013).

32. Hanson, G. W. Drift-diffusion: a model for teaching spatial-dispersion concepts and the importance of screening in nanoscale structures. IEEE Antennas Propag. Mag. 52, 198-207 (2010).

33. Sondheimer, E. H. The mean free path of electrons in metals. Adv. Phys. 1, 1-42 (1952).

34. Ichikawa, M. Theory of localized plasmons for metal nanostructures in random-phase approximation. J. Phys. Soc. Jpn 80, 044606 (2011).

35. Li, X., Xiao, D. \& Zhang, Z. Landau damping of quantum plasmons in metal nanostructures. New J. Phys. 15, 023011 (2013).

36. Andersen, K., Jensen, K. L., Mortensen, N. A. \& Thygesen, K. S. Visualizing hybridized quantum plasmons in coupled nanowires: From classical to tunneling regime. Phys. Rev. B 87, 235433 (2013).

37. Kreibig, U. \& Fragstein, C. The limitation of electron mean free path in small silver particles. Z. Physik. 224, 307-323 (1969).

38. Kreibig, U. \& Vollmer, M. Optical Properties of Metal Clusters (Springer-Verlag, 1995).

39. Gaudry, M. et al. Size and composition dependence in the optical properties of mixed (transition metal/noble metal) embedded clusters. Phys. Rev. B 67, 155409 (2003).

40. Scaffardi, L. \& Tocho, J. Size dependence of refractive index of gold nanoparticles. Nanotechnology 17, 1309-1315 (2006).

41. Kolwas, K. \& Derkachova, A. Damping rates of surface plasmons for particles of size from nano- to micrometers; reduction of the nonradiative decay. J. Quant. Spectrosc. Radiat. Transfer 114, 45-55 (2013). 
42. Nicoletti, O. et al. Surface plasmon modes of a single silver nanorod: an electron energy loss study. Opt. Express 19, 15371-15379 (2011).

43. Wiener, A. et al. Electron-energy loss study of nonlocal effects in connected plasmonic nanoprisms. ACS Nano 7, 6287-6296 (2013).

44. Zhang, S. et al. Experimental demonstration of near-infrared negative-index metamaterials. Phys. Rev. Lett. 95, 137404 (2005).

45. Liu, N., Mesch, M., Weiss, T., Hentschel, M. \& Giessen, H. Infrared perfect absorber and its application as plasmonic sensor. Nano Lett. 10, 2342-2348 (2010).

46. Pors, A., Nielsen, M. G. \& Bozhevolnyi, S. I. Broadband plasmonic half-wave plates in reflection. Opt. Lett. 38, 513-515 (2013).

47. Prodan, E., Radloff, C., Halas, N. \& Nordlander, P. A hybridization model for the plasmon response of complex nanostructures. Science 302, 419-422 (2003).

48. Stella, L., Zhang, P., García-Vidal, F. J., Rubio, A. \& García-González, P. Performance of nonlocal optics when applied to plasmonic nanostructures. J. Phys. Chem. C 117, 8941-8949 (2013).

49. Teperik, T. V., Nordlander, P., Aizpurua, J. \& Borisov, A. G. Robust subnanometric plasmon ruler by rescaling of the nonlocal optical response. Phys. Rev. Lett. 110, 263901 (2013).

50. Teperik, T. V., Nordlander, P., Aizpurua, J. \& Borisov, A. G. Quantum effects and nonlocality in strongly coupled plasmonic nanowire dimers. Opt. Express 21, 27306-27325 (2013).

51. Kadkhodazadeh, S., Wagner, J. B., Kneipp, H. \& Kneipp, K. Coexistence of classical and quantum plasmonics in large plasmonic structures with subnanometer gaps. Appl. Phys. Lett. 103, 083103 (2013).

52. Esteban, R., Borisov, A. G., Nordlander, P. \& Aizpurua, J. Bridging quantum and classical plasmonics with a quantum-corrected model. Nat. Commun. 3, 825 (2012).

53. Ward, D. R., Hüser, F., Pauly, F., Carlos Cuevas, J. \& Natelson, D. Optical rectification and field enhancement in a plasmonic nanogap. Nat. Nanotechnol. 5, 732-736 (2010)

54. Weiss, S., Botkin, D., Ogletree, D. F., Salmeron, M. \& Chemla, D. S. The ultrafast response of a scanning tunneling microscope. Phys. Status Solidi B 188, 343-359 (1995).

55. Grifoni, M. \& Hänggi, P. Driven quantum tunneling. Phys. Rep. 304, 229-354 (1998).

56. Romero, I., Aizpurua, J., Bryant, G. W. \& García de Abajo, F. J. Plasmons in nearly touching metallic nanoparticles: singular response in the limit of touching dimers. Opt. Express 14, 9988-9999 (2006).
57. Fernández-Domínguez, A. I., Wiener, A., García-Vidal, F. J., Maier, S. A. \& Pendry, J. B. Transformation-optics description of nonlocal effects in plasmonic nanostructures. Phys. Rev. Lett. 108, 106802 (2012).

58. Britnell, L. et al. Strong light-matter interactions in heterostructures of atomically thin films. Science 340, 1311-1314 (2013).

59. Koppens, F. H. L., Chang, D. E. \& García de Abajo, F. J. Graphene plasmonics: A platform for strong light-matter interactions. Nano Lett. 11, 3370-3377 (2011).

60. Wang, W. \& Kinaret, J. M. Plasmons in graphene nanoribbons: interband transitions and nonlocal effects. Phys. Rev. B 87, 195424 (2013).

\section{Acknowledgements}

We acknowledge Giuseppe Toscano for use of his finite-element code and we thank Jørn Hvam, Antti-Pekka Jauho and Wei Yan for stimulating discussions. The Center for Nanostructured Graphene (CNG) is funded by the Danish National Research Foundation, Project DNRF58. The A P Møller and Chastine Mc-Kinney Møller Foundation is gratefully acknowledged for the contribution towards the establishment of the Center for Electron Nanoscopy. N.A.M. and M.W. acknowledge financial support by Danish Council for Independent Research-Natural Sciences, Project 1323-00087.

\section{Author contributions}

N.A.M, S.I.B, T.S. and M.W. conceived the basic idea. N.A.M. derived the complex response model. S.R. performed all numerical simulations and the analytical analysis of spherical particles. Figures were prepared by N.A.M. and S.R. All authors interpreted and discussed the results and the writing of the manuscript was done in a joint effort.

\section{Additional information}

Supplementary Information accompanies this paper at http://www.nature.com/ naturecommunications

Competing financial interests: The authors declare no competing financial interests.

Reprints and permission information is available online at http://npg.nature.com/ reprintsandpermissions/

How to cite this article: Mortensen, N. A. et al. A generalized non-local optical response theory for plasmonic nanostructures. Nat. Commun. 5:3809 doi: 10.1038/ncomms4809 (2014) 